\title{
REGISTER RERUM ad Vol. 35
}

Confecit G. Boehm, Basel

Absorption (light), v. Photomicrographs

A cells, v. Pancreas, Pankreas

Achsenskelet und Rückenmark, entwicklungsphysiologische Beziehungen (C) 356

Acini, v. Respiratory tissue

Adrenals, v. Nebenniere, Nebennierenmark

Afferents; on the termination of some afferents to the hippocampus and

fascia dentata (An experimental study in the rat)

202

Age, v. Disques intervertébraux, «Lebenskurve»

Aine, v. Hernies

Allergy, v. Anaphylaktischer Schock

Alter, v. Age

Alveoles, v. Respiratory tissue

Anaphylaktischer Schock, tödlicher, Leberveränderungen (C)

Anatomie; atlas d'anatomie stéréotaxique (Répérage radiologique indirect des noyaux gris centraux des regions mésencéphalo-sous-optique et

hypothalamique de Гhomme) (B) 159

Anatomie chirurgicale des hernies de $\Gamma$ aine (B) $\quad 159$

Anatomische Namen (Ihre Ableitung und Aussprache) (25. Aufl.) (B) . . 160

Anatomy, segmental, of the lungs (B)

Anatomy, v. Microscopic anatomy

Animals, v. Neuropathologie

Anomalie, v. Pancreas

Anus, v. Diplogénèse postérieure

Appareil vestibulaire v. Vestibularapparat

Area entorhinalis, v. Hippocampus

Argent; impregnation à l'argent, résultat obtenus par la technique de Mars-

land, Glees et Erikson (C) 358

-; v. Pancreas

Arterien, v. Papillarmuskelarterien

Articulationes; valeur topographique des plis articulaires et cutanés du

membre inférieur (C) 346

-; v. Gelenkkapsel

Atlas, v. Anatomie

Atrophied muscle fibres, v. Muscle

Auge, v. Tectum opticum

Augenmuskeln; zum Problem der Ringbinden in den Augenmuskeln (C) . . 350

Auris interna, v. Temporal bone, Vestibularapparat

Autonomes Nervensystem, v. Lebístes retL·ulatus 
Aves: carotid body of certain birds 161

Avitaminose (Bj), v. Pankreas

Barrière hémato-endolymphatique, $\mathrm{v}$. Vestibularapparat

Basophilia; on cytoplasmic basophilia in the nerve cells of the cerebral cortex

(I. Methodology) 85

- ; v. Macrophages

1 (B) = Book reviews - Livres nouveaux - Buchbesprechungen

(C) = Report or Demonstration - Communication ou Demonstration - Vortrag

oder Demonstration

Register rerum

361

Bassin, v. Diplogénèse postérieure B cells, v. Pankreas Becken, v. Diplogénèse postérieure

Bindegewebe, v. Connective tissue

Birds; carotid body of certain birds 161

Bleu de trypan, v. Rein

Blood, v. Blutbildung, Blut-Endolympheschranke, Haemoglobin, Hyper-

proteinämie, Pankreaskapillaren, Vessels Blood capillary wall, v. Pankreaskapillaren Blood-

lymph-compartments, v. Vestibularapparat Blutbildung, mesenchymale, im Kopf eines

menschlichen Embryos von 105

$\mathrm{mm} \operatorname{SSL}(\mathrm{C}) \quad 355$

Blut-Endolympheschranke, v. Vestibularapparat

Bone; microscopic anatomy of the temporal bone (B)

Bone; relations between the microscopic structure and tensile strength of

human bone 285

Brain, v. Cerebrum Brechungsindex, v. Refractometry Bronchioli terminales, v. Respiratory

tissue

Canís familíarís; physiologisch-morphologische Untersuchungen über die Innervation des

Nebennierenmarks beim Hund (Veränderungen der Organinnervation in Beziehung zum Alter,

zu experimentellen Einwir-kungen auf den Organismus und zu Krankheiten), Supplementum 33

$(=1$ ad Vol. 35)

-; respiratory tissue of the dog 301

Capillaires sanguins, v. Pankreaskapillaren

Capra, v. Goat, Neurosecretion

Capsula articularis, v. Gelenkkapsel

Caput, v. Blutbildung, Temporal bone

Cardiac muscle, foetal, v. Muscle

Carotid body of certain birds 161

Caryometry, v. Niere, Rein

Cavia porcellus, v. Papillarmuskelarterien

Cells, v. Hyaloplasma

Cerebral cortex; on cytoplasmic basophüia in the nerve cells of the cerebral

cortex (I. Methodology) 85

Cerebrum, v. Cholinestérase, Hippocampus, Hypothalamus, Induction, Stéréotaxique,

Zwischenhirn

Cerveau, v. Cerebrum

Chèvre, v. Goat, Neurosecretion 
Chick embryo; an histological investigation on the epithelioneural bodies in the chick (Gallus dotnesticus) 139

-; v. Tectum opticum

Chien, v. Canis familiaris

Chirurgie, v. Hernies, Stéréotaxique

Choc, v. Schock, Schockbedingungen

Cholinestérase; modifications de $\Gamma$ activité cholinestérasique du tectum

opticum du poulet après destruction de Гébauche de Гæil (C) $\quad 349$

Chorda dorsalis, v. Achsenskelet

362

Register rerum

Chorde encéphaliques, greffons chordencéphaliques; Faction inductrice de la

plaque préchordale sur de $\Gamma$ ectoblaste qui était auparavant influence

par la chorde (Tríturus alpestrís)

47

Chorionepithel, v. Eihautanteile

Ciliary ganglion, v. Ganglions végétatifs céphaliques

Cinématographie (Microcinématographie), v. Neurones

Cobaye, v. Cavía porcellus

Cceur, v. Goat, Papillarmuskelarterien

Collagen fibres, v. Connective tissue fibres

"Colloid" drops, v. "Colloidogenic" function

"Colloidogenic " function of the lung

Coloration, v. Argent, Basophilia, Connective tissue fibres, Pancreas

Columna vertebralis, v. Diplogénèse postérieure, Disques intervertébraux, Halswirbelsäule

Compacta of human bones, v. Bone

Comptes rendus de $\Gamma$ Union Libre des Anatomistes des Universités Suisses,

24e reunion (1958) (C) 344ff.

Connective tissue fibres, unstained, selective demonstration by phase-

contrast refractometry 277

Cor, v. Goat, Papillarmuskelarterien

Coronararterien, v. Papillarmuskelarterien

Corpuscular haemoglobin, v. Haemoglobin

Cortex, v. Cerebral cortex

Cortex renal, v. Rein

Cranium, v. Blutbildung, Temporal bone

Culture de tissus, v. Neurones

Cytochemistry, v. Cholinestérase, "Colloidogenic" function, Histochemische Methoden, Muscle

Cytometry, v. Cytophotometry

Cytophotometry by silver analysis of photomicrographs (Description of a new method and its application to the study of corpuscular haemoglobin), Supplementum 34 (=2 ad. Vol. 35)

Cytoplasma, v. Hyaloplasma

Cytoplasmic basophilia in the nerve cells of the cerebral cortex (I. Methodology) 85

Densitometry, v. Silver analysis

Dental pulp, v. Tooth

Denies, v. Tooth

Dentine, v. Tooth 
Difformité, v. Diplogénèse postérieure, Pancreas

Diffusion, v. Vestibularapparat

Diphtherietoxin, v. Papillarmuskelarterien

Diplogénèse postérieure incomplete chez une femme adulte, un cas 179

Disci intervertebrales, v. Disques intervertébraux, Halswirbelsäule

Discus mandibularis, Strukturanalyse (C) 346

Disques intervertébraux; transformations dans la structure des disques

intervertébraux après la naissance $(\mathrm{C}) \quad 347$

Diverticule du duodenum et persistance des deux ébauches pancréatiques chez

l'adulte (C) 357

Dog, v. Canís famílíaris

Ductus semicirculares, v. Vestibularapparat

Register rerum

363

Duodenum; diverticule du duodenum et persistance des deux ébauches

pancréatiques chez $\Gamma$ adulte (C) $\quad 357$

"Durchdringungsstruktur", v. Hyaloplasma

Ear, v. Temporal bone, Vestibularapparat

Eihautanteile; zur Frage der maternen und fetalen Eihautanteile (C) .... 356

Eínarsori's gallocyanin chrome-alum method, v. Basophilia

Elastic fibres, v. Connective tissue fibres

Elektronenmikroskopische Untersuchungen am exokrinen Pankreas der

weißen Maus. Ultrastruktur der Pankreaskapillaren

63

-; v. Hyaloplasma

Embryo v. Chick embryo, Eihautanteile, Gelenkkapsel, Tectum opticum

Embryo (muscle), v. Muscle

Embryonalhüllen, v. Eihautanteile

Embryonen, menschliche, mit Nackenblasen (C) 356

Endolymphe (Blut-Endolympheschranke), v. Vestibularapparat

Endothel, v. Pankreaskapillaren

Entwicklungsphysiologische Beziehungen zwischen Achsenskelet und

Rückenmark (C) 356

Enzyme reactions, v. Ferments

Epithelioneural bodies in the chick (Gallus domestícus), histological in

vestigation 139

Erikson, v. Marsland

Erythrocytes, v. Haemoglobin

Extremitates, v. Membre inférieur

Eye, v. Augenmuskeln, Tectum opticum

Färbung, v. Argent, Basophilia, Connective tissue fibres, Pancreas

Fascia dentata; on the termination of some afferents to the hippocampus and

fascia dentata (An experimental study in the rat) 202

Feinstruktur des Hyaloplasmas tierischer Zellen (C) 344

Feinstruktur, v. Pankreaskapillaren

Femme adulte, diplogénèse incomplete postérieure, un cas 179

Ferments; some histochemical observations on enzyme reactions in goat 
foetal cardiac muscle and skeletal muscle and some human foetalmuscle 224

-; v. Cholinestérase, Phosphatases

Festigkeit, v. Bone

Fibres of connective tissue, unstained, selective demonstration by phase-

contrast refractometry 277

Fixation, v. Connective tissue fibres

Foetal muscle (cardiac and skeletal muscle), v. Muscle

Foetus; Beitrag zur Kenntnis der Innervation der menschlichen Gelenk

kapsel und der benachbarten Gewebe in der Foetalzeit (C) 355

-; v. Eihautanteile

Foie, v. Leber (...)

Formolschock, v. Mastzellen

Gallocyanin chrome-alum method (Eínarsoń), v. Basophilia

Gallus domestícus, v. Epithelioneural bodies, Tectum opticum

Ganglions végétatifs céphaliques chez le Lebistes reticulatus (Contribution à

l'étude du système nerveux végétatif des poissons osseux II.) 193

,- v. Pneumogastrique

364

Register rerum

Ganglions nerveux sympathiques, modifications au cours du mégacôlon (C) . 348

Gefäße, v. Papillarmuskelarterien, Venae pulmonales

Gehirn, v. Cerebrum

Gelenkkapsel; Beitrag zur Kenntnis der Innervation der menschlichen

Gelenkkapsel und der benachbarten Gewebe in der Foetalzeit (C) . . 355 Génitaux externes, v.

Diplogénèse postérieure Geschlechtschromatin, v. Eihautanteile Gesellschaftsberichte, v.

Verhandlungen ... Glees, v. Marsland Glomus caroticum, v. Carotid body Glottis, v.

Stimmlippen Goat; some histochemical observations on enzyme reactions in goat foetal

cardiac muscle and skeletal muscle and some human foetal muscle . . 224 -; v. Neurosecretion

Gomorí's method (chromic haematoxylin-phloxin method), v. Hypophysis Grafts (material from the presumptive rhombencephalon and spinal cord), $\mathrm{v}$.

Induction Greffons (materiel rhombencéphalique et médullaire présomptif), v. Induction Groin, v. Hernies

Grundlagenforschung, medizinische, Ergebnisse (Bd. 1) (B)

Guinea-pig, v. Cavia porcellus

Haemoglobin; cytophotometry by silver analysis of photomicrographs (Description of a new

method and its application to the study of corpuscular haemoglobin), Supplementum 34 (=2 ad Vol. 35)

Halswirbelsäule, Demonstration zu ihrer «Lebenskurve» (C)

Hauptstücke, v. Niere

Haut, v. Plis articulaires

Heart, v. Goat, Papillarmuskelarterien

Hepar, v. Leber (...)

Heparin, v. "Colloidogenic" function, Mast cells, Mastzellen

Hernies de Гaine, anatomie chirurgicale (B) 159

Herz, v. Goat, Papillarmuskelarterien

Hippocampus; on the termination of some afferents to the hippocampus and 
fascia dentata (An experimental study in the rat) 202

Hî́rschsprung, maladie de Hirschsprung, v. Mégacôlon

Histamine, v. "Colloidogenic" function, Mastzellen

Histochemische Methoden (Lieferung 11-14) (B) 359

Histochemistry, v. Cholinestérase, "Colloidogenic" function, Muscle

Histology, v. Microscopic anatomy

Hormones, v. Hypophysis, Nebenniere

Hühnchen (Embryo), v. Tectum opticum

Human bone, tensile strength, v. Bone

Human foetal muscle, v. Muscle

Human muscle, v. Muscle

Hund; physiologisch-morphologische Untersucliungen über die Innervation des

Nebennierenmarks beim Hund (Veränderungen der Organinnerva-tion in Beziehung zum Alter, zu experimentellen Einwirkungen auf den Organismus und zu Krankheiten), Supplementum 33

$(=1 \mathrm{ad}$ Vol. 35)

Hund; v. Canis familiatis

Register rerum

365

Hyaloplasma tierischer Zellen, Feinstruktur (C) 344

Hyaluronic acid, v. "Colloidogenic" function

Hyperproteinämie; Leber der Maus bei experimenteller Hyperproteinämie (C) 353

Hypophysis; observations on the hypothalamo-hypophysial neurosecretory

system and on variations in the structure of the pituitary of the goat . 1

Hypothalamus; observations on the hypothalamo-hypophysial neurosecretory

system and on variations in the structure of the pituitary of the goat . 1

-; v. Stéréotaxique

Impregnation à l'argent, résultats obtenus par la technique de Marsland,

Glees et Erikson (C) 358

; v. Pancreas

Induction; Faction inductrice de la plaque préchordale sur de $\Gamma$ ectoblaste

qui était auparavant influence par la chorde ( Tríturus alpestris) ... 47

Innervation der menschlichen Gelenkkapsel und der benachbarten Gewebe in

der Foetalzeit (C) 355

Innervation des Nebennierenmarks beim Hund, physiologisch-morpho-logische Untersuchungen

(Veränderungen der Organinnervation in Be-ziehung zum Alter, zu experimentellen

Einwirkungen auf den Orga-nismus und zu Krankheiten), Supplementum 33 (=1 ad Vol. 35)

Innervation, $v$. Tooth

Inseln, v. Pankreas

Intestina, v. Nerf pneumogastrique

Kapillaren, v. Pankreaskapillaren

Karyometrie, v. Niere, Rein

Kidney, v. Niere, Rein

Knochen, v. Bone

Knochenfische, v. Lebístes retL·ulatus

Kohń's paraganglia, v. Epithelioneural bodies

Kollagen, v. Collagen 
Kolloidbildung, v. "Colloidogenic" function

Koronargefäße, v. Papillarmuskelarterien

Krankheiten, v. Nebennierenmark

Labrocytes, v. Mastzellen

Labyrinth, v. Vestibularapparat

Langer $1 / 8 \alpha$ ns'sche Inseln, v. Pancreas, Pankreas

Larves de Triturus alpestris (porteuses des greffons chordencéphaliques), v. Induction

«Lebenskurve» der Halswirbelsäule (C) 347

Leber der Maus bei experimenteller Hyperproteinämie (C) 353

Leberveränderungen bei tödlichem anaphylaktischem Schock (C) 353

Lebistes retículatus; la branche intestinale du nerf pneumogastrique chez

Lebistes retículatus (Contribution à $\Gamma$ étude du système nerveux végétatif

des poissons osseux I.)

; les ganglions végétatifs céphaliques chez le Lebistes reticulatus (Con

tribution à Гétude du système nerveux végétatif des poissons osseux II.) 193

Leistenregion, v. Hernies

Lipoproteins, v. "Colloidogenic" function

Acta anat., Vol. 35, No. 4 (1958)

24

366

Register rerum

Liver, v. Leber (...)

Lung; "colloidogenic" function $\quad 310$

-; respiratory tissue of the dog 301

-; segmental anatomy of the lungs (B) $\quad 254$

-; v. Pulmonalvenen, Pulmonary macrophages

Macrophages; experimental transformation of pulmonary macrophages into

cells displaying the aspect of mast cells 215

Macrophages, pulmonary, v. "Colloidogenic" function

Malformation, v. Diplogénèse postérieure, Pancreas

Mammalia, v. Respiratory tissue, Vestibularapparat

Man, v. Anatomy, Augenmuskeln, Bone, Diplogénèse postérieure, Hernies,

Leberveränderungen, Muscle, Nackenblasen, Neuropathologie, Niere, Pancreas, Stéréotaxique,

Stimmlippen, Venae pulmonales, Vestibularapparat

Mandibula, v. Discus mandibularis

Marsland, Glees et Eríkson, v. Impregnation à l'argent

Mast cells; experimental transformation of pulmonary macrophages into

cells displaying the aspect of mast cells

215

Mastzellen, Einfluß experimenteller Schockbedingungen 118

Maus, v. Mus musculus

Mechanoreceptors, v. Tooth

Medizinische Grundlagenforschung, Ergebnisse (Bd. 1) (B)

255

Medulla, v. Induction

Medulla spinalis, v. Rückenmark

Meerschweinchen, v. Cavia porcellus

Mégacôlon; modifications des ganglions nerveux sympathiques au cours du 
mégacôlon (C) 348

Mégadolycôlon, v. Mégacôlon

Membrane semiperméable ultrafiltrante, v. Vestibularapparat

Membre inférieur; valeur topographique des plis articulaires et cutanés du membre inférieur (C) 346

Menschliche Embryonen mit Nackenblasen (C) 356

Mesencephalon, v. Stéréotaxique, Zwischenhirn

Mesenchymale Blutbildung im Kopf eines menschliche Embryos von 105 mm

SSL(C) $\quad 355$

Metachromasie, v. Macrophages

Methode, v. Basophilia, Histochemische Methoden, Impregnation à l'argent, Pancreas, Silver analysis

Microcinématographie, v. Neurones

Microphotometry, v. Basophilia

Microscopic anatomy of the temporal bone (B) 359

Microscopic structure and tensile strength of human bone, relations .... 285

Mißbildung, v. Diplogénèse postérieure, Pancreas

Mittelstücke, v. Niere

Moelle épinière, v. Induction, Rückenmark

Monstruosité, v. Diplogénèse postérieure

Motor end-plates, v. Ferments ...

Mouse, v. Mus musculus

Mucopolysaccharides, v. "Colloidogenic" function

Register rerum

367

Mus musculus, v. Achsenskelet, Hyperproteinämie, Pankreaskapillaren, Rein Muscle; acid and alkaline phosphatases in normal and diseased human muscle 326 --; some histochemical

observations on enzyme reactions in goat foetal

cardiac and skeletal muscle and some human foetal muscle 224

-; v. Augenmuskeln

Muscles pectoraux, nerfs (B) 253

Muscular atrophy, v. Muscle

Muscular dystrophy, v. Muscle

Musculi pectorales, v. Muscles pectoraux

Musculo-tendinous junctions, v. Muscle

Musculus peronaeus longus, Fixation seiner Sehne, und Os peronaeum (C) . 345

Muskel, v. Muscle

Myofibrillen, v. Ringbinden

Myokard, v. Papillarmuskelarterien

Nackenblasen; menschliche Embryonen mit Nackenblasen (C) $\quad 356$

Namen, anatomische (Ihre Ableitung und Aussprache), (25. Auf1.) (B) . 160

Nebenniere, Einfluß auf die Zellkerngröße der Nierentubuli (C) 352

Nebennierenmark beim Hund, physiologisch-morphologische Untersuchun-gen über dessen Innervation (Veränderungen der Organinnervation in Beziehung zum Alter, zu experimentellen Bedingungen auf den Orga-nismus und zu Krankheiten), Supplementum 33 (=1 ad Vol. 35) 
Nerf pneumogastrique, branche intestinale, chez Lebistes retículatus (Contribution à $\Gamma$ étude du système nerveux végétatif des poissons osseux I.) 132

Nerfs des muscles pectoraux (B) 253

Nerve cells; on cytoplasmic basophilia in the nerve cells of cerebral cortex

(I. Methodology) 85

Nerven (Nervensystem, Zentralnervensystem), v. Afferents, Cholinestérase, Epithelioneural bodies, Ferments ... ., Ganglions végétatifs céphaliques, Gelenkkapsel, Induction, Mégacôlon, Nebennierenmark, Nerf(s), Nerve cells, Neurones, Neuropathologie,Neurosecretion,

Stéréotaxique, Subkommissurales Organ, Tooth

Nervus sympathicus, v. Sympathicus

Nervus vagus, v. Lebistes reticulatus

Neurochirurgie, v. Stéréotaxique

Neurofibrilles, v. Neurones

Neuro-hepatic complexes, v. Epithelioneural bodies

Neurohypophyse, v. Hypophysis

Neurones, structure "in vivo" (C) 349

Neuropathologie, vergleichende, des Menschen und der Tiere (B) 254

Neurosecretion; observations on the hypothalamo-hypophysial neurosecretory

system and on variations in the structure of the pituitary of the goat 1

Neurulas de Triturus alpestris, v. Induction

Neurulation, v. Induction

Niere; Einfluß der Nebenniere auf die Zellkerngröße der Nierentubuli (C) . . 352

-; Zellkerngröße der Haupt- und Mittelstücke menschlicher Nieren (C) . . 351

$-; \mathrm{v}$. Rein

Nierentubuli, v. Niere

Nomenklatur, v. Anatomische Namen

Nomina anatomica, v. Anatomische Namen

368

Register rerum

Noyaux, v. Rein, Sex chromatin, Zellkerngröße

Nucléaire; gonflement nucléaire fonctionnel dans les reins de souris après in

jection de bleu de trypan et de Tyrode (C) 352

-; taille nucléaire des segments initiaux et intermédiaires des regions

souscapsulaires et juxtamédullaires du rein de rat (C) 351

-; v. Zellkerngröße

Nuclei, v. Rein, Sex chromatin, Zellkerngröße

Nucleic acids, v. Basophilia

Oculus, v. Augenmuskeln, Tectum opticum

Oeil, v. Augenmuskeln, Tectum opticum

Ohr, v. Temporal bone, Vestibularapparat

Oiseaux, v. Carotid body

Ontogenesis, v. Blutbildung, Chick embryo, Diplogénèse postérieure, Eihaut-

anteile, Gelenkkapsel, Induction, Nackenblasen, Pancreas, Rücken-

mark Oreille, v. Temporal bone, Vestibularapparat Organum subcommissurale, v.

Subkommissurales Organ Os, v. Bone Os peronaeum; Fixation der Sehne des M. peronaeus

longus und Os peronaeum 
(C) 345

Os sacrum, v. Diplogénèse postérieure

Os temporale; microscopic anatomy of the temporal bone (B)

Osteones, v. Bone Otology, v. Temporal bone

Pancreas; diverticule du duodenum et persistance des deux ébauches pan-

créatiques chez Tadulte (C) 357

-; nouvelle méthode de mise en evidence des cellules A du pancreas . . 107

-; v. Pankreas

Pancreatic islets, v. Pancreas, Pankreas

Pankreas; Größe und Funktionsindex der Inseln Bj-avitaminotischer Ratten

(C) 354

-; v. Pancreas

Pankreaskapillaren, Ultrastruktur (Elektronenmikroskopische Unter-

suchungen am exokrinen Pankreas der weißen Maus)

- ; v. Pancreas

Papillarmuskelarterien des Meerschweinchens, experimentelle Strukturver-

änderungen nach Diphtherietoxin (C) 357

Paraganglia (Kohrí's paraganglia; abdominal vagal paraganglia), v. Epithe-

lioneural bodies " Paraganglion caroticum", v. Carotid body Parasymphaticus, v. Ganglions

végétatifs céphaliques Pathological human muscle, v. Muscle Pathologie, v. Nebennierenmark, Neuropathologie Peau, v. Plis articulaires

Pectoraux; muscles pectoraux, nerfs (B)

253

Pelvis, v. Diplogénèse postérieure Peptonschock, v. Mastzellen Periodontium, v. Tooth

Register rerum

369

Permeabilität, v. Vestibularapparat

Phase-contrast microscopy, v. Neurones

Phase-contrast refractometry; selective demonstration of unstained connec

tive tissue fibres by phase-contrast refractometry 277

Phosphatases, acid and alkaline, in normal and diseased human muscle . . . 326

Photographic density, v. Photomicrographs

Photometry, v. Basophilia, Photomicrographs

Photomicrographs; cytophotometry by silver analysis of photomicrographs (Description of a new method and its application to the study of corpuscular haemoglobin), Supplementum 34 (=2 ad Vol. 35)

Pisces, v. Lebístes reticulatus

Pituitary, v. Hypophysis

Plaque préchordale; Faction inductrice de la plaque préchordale sur de l'ecto-

blaste qui était auparavant influence par la chorde (Tríturus alpestris) 47

Plicae vocales, v. Stimmlippen

Plis articulaires et cutanés du membre inférieur, valeur topographique (C) . 346

Pneumoconioses, v. " Colloidogenic" function

Pneumogastrique, nerf -; la branche intestinale du nerf pneumogastrique

chez Lebístes reticulatus (Contribution à $\Gamma$ étude du système nerveux

végétatif des poissons osseux I.) 132

-; v. Ganglions végétatifs 
Poissons osseux, v. Lebístes reticulatus

Poulet, v. Tectum opticum

Poumon, v. "Colloidogenic" function, Pulmo, Pulmonary macrophages, Respiratory tissue,

Venae pulmonales

Poussin, v. Tectum opticum

Prächordale Platte, v. Induction

Prechordal plate, v. Induction

Processus uncinati, v. Halswirbelsäule

Proteins, v. Hyperproteinämie

Pulmo; segmental anatomy of the lungs (B) 254

-; v. "Colloidogenic" function, Pulmonary macrophages, Respiratory tissue, Venae pulmonales

Pulmonary macrophages; experimental transformation of pulmonary

macrophages into cells displaying the aspect of mast cells 215

Pulmonalvenen, v. Venae pulmonales

Radiologie, v. Stéréotaxique

Rat, v. Hippocampus, Mastzellen, Pankreas, Rein

Rayons X, v. Stéréotaxique

Receptors of the tooth, morphology

257

Rectum, v. Diplogénèse postérieure

Refractometry; selective demonstration of unstained connective tissue

fibres by phase-contrast refractometry

277

Regio inguinalis, v. Hernies

Rein; a propos du gonflement nucléaire fonctionnel dans les reins de souris

après injection de bleu trypan et de Tyrode $(\mathrm{C}) \quad 352$

-; de la taille nucléaire des segments initiaux et intermédiaires des

regions souscapsulaires et juxtamédullaires du rein de rat (C) .... $351-$; v. Zellkerngröße

370

Register rerum

Ren, v. Niere, Rein

Resistance à la traction, $v$. Bone

Respiratory tissue of the dog 301

Reticular fibres, v. Connective tissue fibres Reticulum, v. Hyaloplasma Rhombencephalon, v.

Induction

Ringbinden in den Augenmuskeln, zu deren Problem (C) 350

Röntgenaufnahmen, v. Stéréotaxique

Rückenmark und Achsenskelet, entwicklungsphysiologische Beziehungen (C) 356

-; v. Induction

Schock, anaphylaktischer, tödlicher, Leberveränderungen (C) 353

Schockbedingungen, experimentelle, Einfluß auf die Mastzellen 118

Schweiz, v. Verhandlungen ...

Sclerosis, pulmonary sclerosis, v. " Colloidogenic" function

Segmental anatomy of lungs (B) 254

Segmentgrenzen, v. Venae pulmonales

Segments initiaux et intermédiaires des regions souscapsulaires et juxta-

médullaires du rein de rat, taille nucléaire $(\mathrm{C}) \quad 351$

Sehnen; Muskel-Sehnen-Übergänge, v. Ferments ... 
Semicircular tubes, v. Vestibularapparat

"Semipermeable Ultrafilter-Membran", v. Vestibularapparat

Sensory nerve endings, v. Tooth

Sex chromatin, v. Eihautanteile

Shock, v. Schock, Schockbedingungen

Silver analysis of photomicrographs; cytophotometry by silver analysis of photomicrographs

(Description of a new method and its application to the study of corpuscular haemoglobin),

Supplementum 34 (=2 ad Vol. 35)

Silver impregnation, v. Impregnation à $\Gamma$ argent, Pancreas

Skelet, v. Achsenskelet

Skeletal muscle, v. Muscle

Skeletal muscle, foetal, v. Goat

Skin, v. Plis articulaires

Sociétés, v. Comptes rendus ...

Society transactions, v. Verhandlungen ... .

Souris, v. Mus musculus

Spannmechanismus der menschlichen Stimmlippen, neue Beiträge zu dieser

Frage $(C) \quad 345$

Spinal cord, v. Induction

Staining, v. Argent, Basophilia, Connective tissue fibres, Pancreas

Statistische Auswertung, v. Niere, Rein

Stéréotaxique; atlas d'anatomie stéréotaxique (Repérage radiologique in

direct des noyaux gris centraux des regions mésencéphalo-sous-optique

et hypothalamique de Thomme) (B) 159

Stimmlippen, menschliche, neue Beiträge zu ihrem Spannmechanismus (C) . 345

Strength; tensile strength and microscopic structure of human bone, relations 285

Subkommissurales Organ des Zwischenhirns (C) $\quad 348$

Submicroscopical structure, v. Hyaloplasma, Pankreaskapillaren

Sub-neural apparatus, v. Ferments ...

Suisse, v. Comptes rendus ... .

Register rerum

371

Surrénales, v. Nebenniere, Nebennierenmark

Sympathicus; modifications des ganglions nerveux sympathiques au cours

du mégacôlon (C) $\quad 348$

-; v. Ganglions végétatifs céphaliques

Système nerveux végétatif, v. Ganglions végétatifs céphaliques, Nerf pneu-

mogastrique

Tectum opticum; modifications de Tactivité cholinestérasique du tectum

opticum du poulet après destruction de Гébauche de Гceil (C) .... 349 Teeth, v. Tooth

Temporal bone, microscopic anatomy (B) 359

Tendinous; musculo-tendinous junctions, v. Ferments ...

Tensile strength and microscopic structure of human bone, relations .... 285

Teratologie, v. Diplogénèse postérieure, Pancreas

Tiere, v. Neuropathologie

Tissu conjonctif, v. Connective tissue 
Tissue cultures, v. Neurones

Titration, v. Silver analysis

Tooth; morphology of receptors of the tooth 257

Topographie, v. Plis artuculaires

Toxin (Diphtherietoxin), v. Papillarmuskelarterien

Traction, resistance à la traction, v. Bone

Transmission, v. Silver analysis

Transplantation, v. Induction

Tríturus alpestris, v. Induction

Trypanblau, v. Rein

Tubuli, v. Niere

Tyrodelösung, v. Rein

Ultrastruktur der Pankreaskapillaren (Elektronenmikroskopische Unter-

suchungen am exokrinen Pankreas der weißen Maus) 63

-; v. Hyaloplasma

Unstained connective tissue fibres, selective demonstration by phase-contrast

refractometry 277

Uterus, v. Diplogénèse postérieure

Vagal paraganglia, abdominal, v. Epithelioneural bodies

Vagina, v. Diplogénèse postérieure

Vagus, v. Ganglions végétatifs, Nerf pneumogastrique

Vaisseaux, v. Papillarmuskularterien, Venae pulmonales

Variationen, v. Venae pulmonales

Végétatifs; ganglions végétatifs céphaliques chez le Lebistes retículatus (Contribution à Гétude

du système nerveux végétatif des poissons osseux II.) 193

-; v. Pneumogastrique

Veins, v. Venae pulmonales

Venae pulmonales, Variationen ihrer Sammelgebiete, Zusammenhang mit

den Segmentgrenzen $(\mathrm{C}) \quad 345$

Venen, $v$. Venae pulmonales

Vergleichende Neuropathologie des Menschen und der Tiere (B) 254

Verhandlungen der Freien Vereinigung der Anatomen an Schweizerischen

Hochschulen, 24. Tagung (1958) (C) 344ÍF.

Versilberung, v. Impregnation à $\Gamma$ argent

372

Register rerum

Vertebrae cervicales, v. Halswirbelsäule

Vertebrae lumbales (Vertebrae sacrales), v. Diplogénèse postérieure

Vesica urinaria, v. Diplogénèse postérieure

Vessels, v. Papillarmuskelarterien, Venae pulmonales

Vestibularapparat bei Mensch und Säugetieren, eine dort vorkommende

besondere Gewebsart

15

Vitalfärbung, v. Vestibularapparat Vitamin B15 v. Pankreas Vögel, v. Carotid body Vulva, v.

Diplogénèse postérieure

Wärmeschock, v. Mastzellen

$\mathrm{X}$-rays, v. Stéréotaxique 
Zähne, v. Tooth

Zellen, v. Hyaloplasma

Zellkerngröße der Haupt- und Mittelstücke menschlicher Nieren (C) .... 351

Zellkerngröße der Nierentubuli, Einfluß der Nebenniere (C) 352

$-; \mathrm{v}$. Rein

Ziege, v. Goat, Neurosecretion

Zugfestigkeit, v. Bone

Zwischenhirn, subkommissurales Organ (C) 348

Zyto ..., v. Cyto ...

News from Universities - Nouvelles universitaires - Universítätsnachrichten

Bujard, E 256

Rouiiler, Ch 256

Theiler, K 256

Book Reviews - Livres Nouveaux - Buchbesprechungen

Bauer, K. (Editor): Ergebnisse der medizinischeii Grundlageiiforschung

(Bd. I) (G. Thieme, Stuttgart 1956) 255

Boyden, E. A.: Segmental anatomy of the lungs (McGraw-Hill Book Comp., Inc., New York/Toronto/London 1955) 254

Frauchiger, E. und R. Fankhauser: Vergleichende Neuropathologie des

Menschen und der Tiere (Springer Verlag, Berlin/Göttingen/Heidelberg 1957) 254

Fruchaud, H.: Anatomie chirurgicale des hernies de Гaine (G. Doin \& Cie., Paris 1956) 159

Lipp, W.: Histochemische Methoden (Lieferungen 11-14) (R. Oldenbourg, München 1957) 359

Paturet, J.: Les nerfs des muscles pectoraux (Impr. R. Foulon, Paris 1957) . 253

Talaírach, J., M. David, P. Tournoux, H. Corredor et T. Kvasína: Atlas

d'anatomie stéréotaxique (Repérage radiologique indirect des noyaux gris centraux des regions mésencéphalo-sous-optique et hypothalamique de Thomme) (Masson \& Cie., Paris 1957) 159

Triepel, H./Herrlínger, R.: Die anatomischen Namen (25. Aufl.) (J.F. Bergmann, München 1957) 160

Wolff, D., R. J. Belluci and A. A. Eggston: Microscopic anatomy of the temporal bone (The Williams \& Wilkins Co., Baltimore 1957), ... 359 